A high sensitivity nanomaterial based SAW humidity sensor

This article has been downloaded from IOPscience. Please scroll down to see the full text article.

2008 J. Phys. D: Appl. Phys. 41085101

(http://iopscience.iop.org/0022-3727/41/8/085101)

The Table of Contents and more related content is available

Download details:

IP Address: 140.112.113.225

The article was downloaded on 23/12/2008 at 02:31

Please note that terms and conditions apply. 


\title{
A high sensitivity nanomaterial based SAW humidity sensor
}

\author{
Tsung-Tsong Wu ${ }^{1,3}$, Yung-Yu Chen ${ }^{2}$ and Tai-Hsu Chou ${ }^{1}$ \\ ${ }^{1}$ Institute of Applied Mechanics, National Taiwan University, Taipei 106, Taiwan, Republic of China \\ ${ }^{2}$ Department of Mechanical Engineering, Tatung University, Taipei 104, Taiwan, Republic of China \\ E-mail: wutt@ndt.iam.ntu.edu.tw
}

Received 9 October 2007, in final form 4 February 2008

Published 5 March 2008

Online at stacks.iop.org/JPhysD/41/085101

\begin{abstract}
In this paper, a highly sensitive humidity sensor is reported. The humidity sensor is configured by a $128^{\circ} \mathrm{YX}-\mathrm{LiNbO}_{3}$ based surface acoustic wave (SAW) resonator whose operating frequency is at $145 \mathrm{MHz}$. A dual delay line configuration is realized to eliminate external temperature fluctuations. Moreover, for nanostructured materials possessing high surface-to-volume ratio, large penetration depth and fast charge diffusion rate, camphor sulfonic acid doped polyaniline (PANI) nanofibres are synthesized by the interfacial polymerization method and further deposited on the SAW resonator as selective coating to enhance sensitivity. The humidity sensor is used to measure various relative humidities in the range $5-90 \%$ at room temperature. Results show that the PANI nanofibre based SAW humidity sensor exhibits excellent sensitivity and short-term repeatability.

(Some figures in this article are in colour only in the electronic version)
\end{abstract}

Over the past few decades, a number of research works [13 ] focused on surface acoustic wave (SAW) devices because of their small size, low cost and capability of compatibility with the integrated circuit process. Moreover, SAWs are sensitive to surface perturbation such as mass loading, viscoelastic change, or electrical alternation for acoustic energy is confined within one or two wavelengths near the surface. This feature also contributes to high stability, short response time and good reproducibility. Due to their remarkable characteristics, SAW devices are very suitable for application to real-time sensors. For humidity detection, hygroscopic selective coating must be deposited on the sensors. There are many investigations focused on the sensing characteristics of hygroscopic materials (mainly polymer films) [4-10]. Although polymer films can offer a feasible way of grabbing water molecules, there is still a detection limitation on account of less interaction area. Even porous hygroscopic films cannot noticeably improve the sensing efficiency because of the intrinsic restriction of the exposure area. On the other hand, nanostructures such as nanorods, nanobelts and nanofibres possess high surfaceto-volume ratio, large penetration depth and fast charge diffusion rate and have been utilized by combining with

\footnotetext{
3 Author to whom any correspondence should be addressed.
}

SAW sensors for gas sensing such as $\mathrm{H}_{2}, \mathrm{CO}, \mathrm{NO}_{2}$ and volatile organic compounds [11-13]. However, to the best of our knowledge, SAW sensors with nanostructured selective coatings for humidity detection have not yet been found.

In this paper, we proposed a high sensitivity humidity sensor by utilizing polyaniline (PANI) nanofibres as the selective coating for relative humidity $(\mathrm{RH})$ detection. A dual delay line system [7,14], which consisted of two counterparts in oscillator where one was coated with nanostructured selective coating and the other was bare to execute common mode rejection, was realized to eliminate external temperature fluctuations. Several experimental tests were engaged in gathering the RH sensing characteristics of this system at room temperature, inclusive of temperature effect, sensitivity and repeatability.

First of all, the coupling-of-modes model was utilized to predict device performances of the two-port SAW resonators prior to fabrication. Then, the SAW resonators were fabricated by the micro-electro-mechanical system process: interdigital transducers (IDTs) and reflectors were patterned by exposure and development after the aluminium film was deposited on a $128^{\circ} \mathrm{YX}-\mathrm{LiNbO}_{3}$ substrate. The wavelength, centre frequency and the delay line are $27 \mu \mathrm{m}, 145 \mathrm{MHz}$ and 


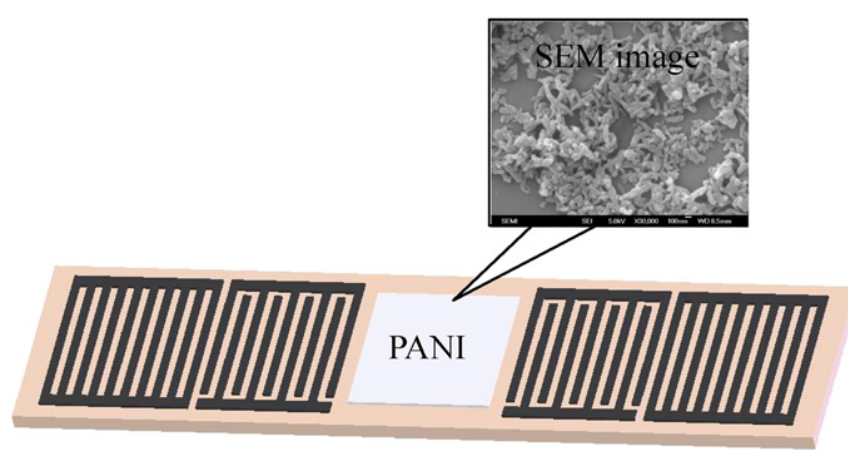

Figure 1. Schematic diagram of a PANI nanofibre based SAW resonator and SEM image of the PANI nanofibres.

$4320 \mu \mathrm{m}$, respectively. The number of electrode pairs of IDTs is 15 , while the electrode number of the reflectors is 50 .

The camphor sulfonic acid (CSA) doped PANI nanofibres were synthesized by the interfacial polymerization method. The synthesis steps are stated as follows: first, 1 mmole of ammonium peroxydisulfate was added to $1 \mathrm{M} \mathrm{CSA}$ in $10 \mathrm{ml}$, and then 4 mmole aniline monomer was dissolved in $10 \mathrm{ml}$ hexane organic phase. The two aqueous solutions in a glass vial were mixed uniformly, and consequently interfacial polymerization occurred. The mixed solution was placed overnight to make the reaction complete. To remove redundant acid and by-products, the solution was washed with deionized water and purified by an air-extracting apparatus equipped with a $0.2 \mu \mathrm{m}$ filter paper. After drying in atmosphere, dark green granular PANI nanofibres were obtained. The scanning electron microscope (SEM) image of the PANI nanofibres shown in figure 1 revealed a large quantity of wire-like nanostructures. Finally, the nanofibres were dissolved in $3 \mathrm{ml}$ deionized water. A micropipette with 5-50 $\mu$ l capacity was adopted to spread a $5 \mu$ l solution onto the sensing area of the SAW resonator, and then the resonator was left to dry in a clean and dry environment.

In order to enhance the sensing stability, constructing a dual delay line SAW sensor system is imperative for eliminating the noises from ambient temperature and pressure. In general, the dual delay line system consists of two identical amplifiers, a mixer and two comparative SAW resonators. The oscillation was obtained by using the topology of a closeloop which consisted of an active amplifier and a frequencysensitive feedback resonator. In addition, one of the resonators was coated with PANI nanofibres to serve as a sensor, and the other was bare regarded as a reference.

The sensing system was mounted in an acrylic chamber with a volume of about $350 \mathrm{~cm}^{3}$. The chamber was drilled for the sake of wire link, gas entrance and power supply. Furthermore, a two-way joint was utilized to mix water vapour with nitrogen as the carrier for altering the $\mathrm{RH}$ inside the chamber. A flow meter with $1-5 \mathrm{~L} \mathrm{~min}^{-1}$ flow rate, which was laid between the joint and the chamber, provided pressure stability. The RH inside the chamber was monitored by a commercial transmitter, KIMO TH100, which has an accuracy of $\pm 3 \%$ with respect to the RH range of $0-100 \%$. Through the integration of the components mentioned above, a gas flow system was successfully constructed; moreover, the

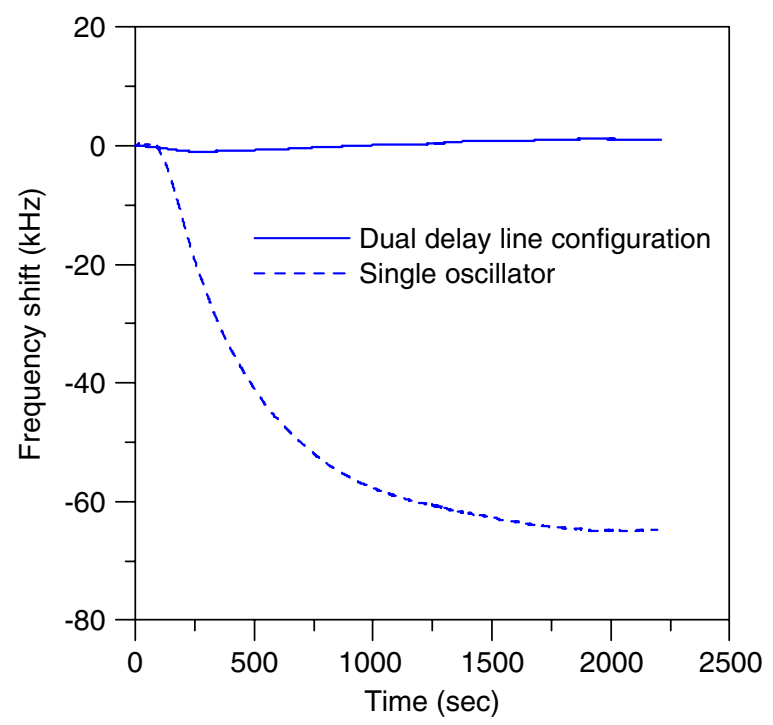

Figure 2. Thermal tests of a single oscillator and a dual delay line configuration.

differential frequency was read by a programmable frequency counter. A laptop was used to real-time access data from the counter through the general purpose interface bus (GPIB).

Because the temperature coefficient of delay (TCD) of $128^{\circ} \mathrm{YX}-\mathrm{LiNbO}_{3}$ is large, temperature plays the most important role in sensing precision among environmental factors. The substrate has a TCD of $75 \mathrm{ppm}^{\circ} \mathrm{C}^{-1}$, which means that a frequency drift per degree is $11 \mathrm{kHz}$ corresponding to the $27 \mu \mathrm{m}$ wavelength. Therefore, a thermal test was implemented to observe the response towards a $5{ }^{\circ} \mathrm{C}$ heating. As shown in figure 2 , a $63 \mathrm{kHz}$ frequency change occurred during the temperature variation for the single oscillator. To compensate the temperature effect, we constructed a dual delay line configuration. One more thermal test with the same temperature variation was implemented for this configuration. The maximum frequency change was only $1.5 \mathrm{kHz}$. The dual delay line configuration indeed suppressed the temperature noise and could enhance sensing stability.

Experiments have also been done for investigating the short-term repeatability of the sensor. At the initial stage, the steady state of the baseline frequency was reached, and afterwards nitrogen or mixed water vapour flowed into the chamber to alter the RH inside the chamber. Testing cycles were implemented with constant exposure time and purge time to reach a new steady state or re-establish the baseline. The results shown in figure 3 indicated that the frequency shifts are 60,80 and $110 \mathrm{kHz}$ corresponding to the $\mathrm{RH}$ variation of $20 \%$, $30 \%$ and $45 \%$. These results demonstrated that the proposed sensing system possesses good reaction response and recovery response. Moreover, it is well known that moisture can make PANI more conductive [15-17], which results in a reduction in SAW velocity and thus leads to a frequency decrease. The characteristic also appeared in figure 3.

For the sake of comparing the uncoated oscillator with the coated one, their responses towards a $\mathrm{RH}$ variation were measured at room temperature. Evidently the frequency of the uncoated one did not show an obvious change, and 


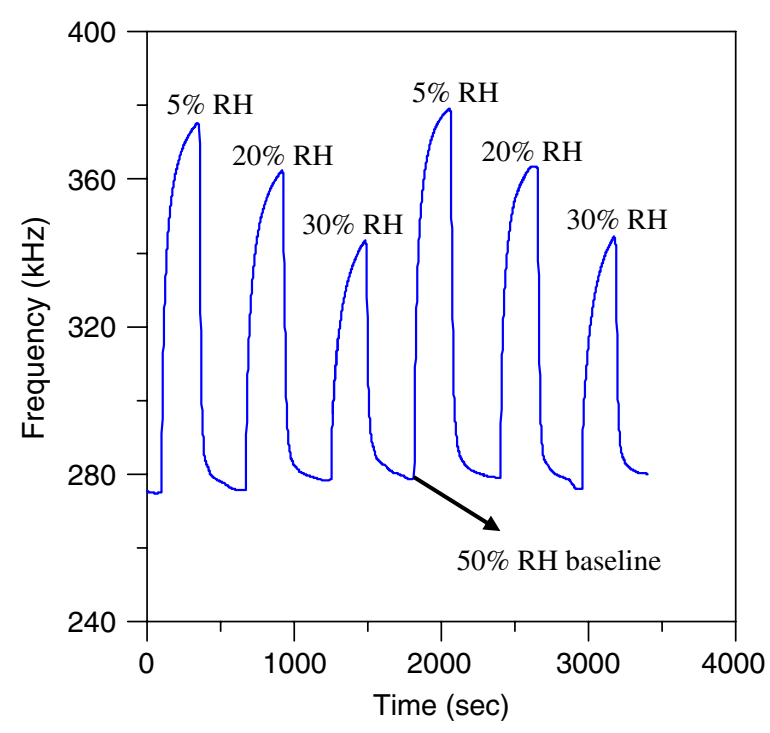

Figure 3. Short-term repeatability of a dual delay line configuration.

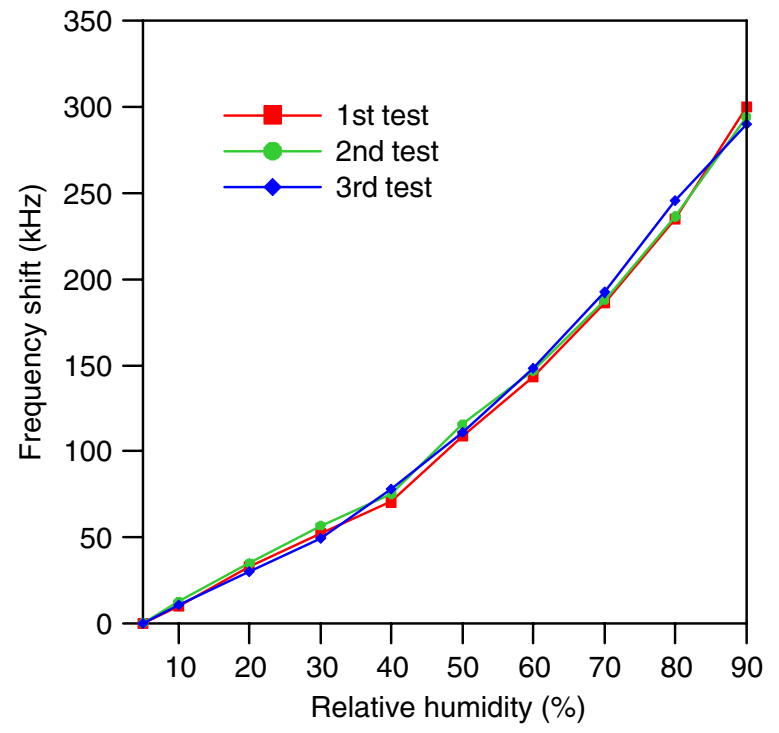

Figure 4. Changes in frequency with relative humidity.

the maximum shift was about $3 \mathrm{kHz}$. In contrast, a larger frequency shift can be easily observed for the oscillator coated with PANI nanofibres. Figure 4 shows the sensitivity of the dual delay line configuration coated with PANI nanofibres. There were 2.45 and $4.58 \mathrm{kHz}$ drop in frequency per cent of $\mathrm{RH}$ variation in the range of $\mathrm{RH}$ below $50 \%$ and above. In terms of $\Delta f / f$, the sensitivity is at least $16.8 \mathrm{ppm} / \% \mathrm{RH}$ at room temperature. This is about twice higher than that in [10] $(8.57 \mathrm{ppm} / \% \mathrm{RH})$, which is the best among the publications about SAW humidity sensors.

In summary, the PANI nanofibres can be applied to form a high sensitivity humidity sensor. By utilizing a dual delay line configuration, the temperature noise can be well suppressed. In the literature, SAW humidity sensors were coated with a traditional hygroscopic film as selective coating. Since nanostructured materials possess high surface-to-volume ratio and fast electron mobility, the PANI nanofibre based SAW humidity sensor indeed possesses high sensitivity and is an excellent candidate for a humidity sensor operating at room temperature.

\section{References}

[1] Howe E and Harding G 2000 A comparison of protocols for the optimization of detection of bacteria using a surface acoustic wave (SAW) biosensor Biosens. Bioelectron. 15 641-9

[2] Jakubik W P, Urbanczyk M W, Kochowski S and Bodzenta J 2002 Bilayer structure for hydrogen detection in a surface acoustic wave sensor system Sensors Actuators B-Chem. 82 265-71

[3] Kondoh J, Muramatsu T, Nakanishi T, Matsui Y and Shiokawa S 2003 Development of practical surface acoustic wave liquid sensing system and its application for measurement of Japanese tea Sensors Actuators B-Chem. 92 191-8

[4] Nomura T, Oofuchi K, Yasuda T and Furukawa S 1994 SAW humidity sensor using dielectric hygroscopic polymer film IEEE Ultrasonics Symp. (Cannes, France) pp 503-6

[5] Braga E R, Nakano A Y and da Cunha M P 1999 A SAW resonator sensor system employed in humidity measurements Proc. SBMOEEE MIT-SIMOC'99 (Rio de Janeiro, Brazil) pp 342-5

[6] Penza M and Anisimkin V I 1999 Surface acoustic wave humidity sensor using polyvinyl-alcohol film Sensors Actuators A-Phys. 76 162-6

[7] Penza M and Cassano G 2000 Relative humidity sensing by PVA-coated dual resonator SAW oscillator Sensors Actuators B-Chem. 68 300-6

[8] Tashtoush N M, Cheeke J D N and Eddy N 1998 Surface acoustic wave humidity sensor based on a thin PolyXIO film Sensors Actuators B-Chem. 49 218-25

[9] Nieuwenhuizen M S and Nederlof A J 1990 A SAW gas sensor for carbon dioxide and water: preliminary experiments Sensors Actuators B-Chem. 297-101

[10] Korsah K, Ma C L and Dress B 1998 Harmonic frequency analysis of SAW resonator chemical sensors: application to the detection of carbon dioxide and humidity Sensors Actuators B-Chem. 50 110-6

[11] Penza M, Antolini F and Vittori Antisari M 2004 Carbon nanotubes as SAW chemical sensors materials Sensors Actuators B-Chem. 100 47-59

[12] Penza M, Antolini F and Vittori Antisari M 2005 Carbon nanotubes-based surface acoustic waves oscillating sensor for vapour detection Thin Solid Films 472 246-52

[13] Sadek A Z, Wlodarski W, Shin K, Kaner R B and Kalantar-Zadeh K 2006 A layered surface acoustic wave gas sensor based on a polyaniline/ $/ \mathrm{In}_{2} \mathrm{O}_{3}$ nanofibre composite Nanotechnology 17 4488-92

[14] Ballantine D S, White R M, Martin S J, Ricco A J, Frye G C, Zellers E T and Wohltjen H 1997 Acoustic Wave Sensors: Theory, Design, and Physico-chemical Application (New York: Academic)

[15] Angelopoulos M, Ray A, MacDiarmid A G and Epstein A J 1987 Polyaniline: processability from aqueous solutions and effect of water vapor on conductivity Synth. Met. $2121-30$

[16] MacDiarmid A G and Epstein A J 1989 Polyanilines: a novel class of conducting polymers Faraday Discuss. Chem. Soc. $88317-32$

[17] Lubentsov B Z, Timofeeva O N and Khidekel M L 1991 Conducting polymer interaction with gaseous substances: II. PANI- $\mathrm{H}_{2} \mathrm{O}, \mathrm{PANI}-\mathrm{NH}_{3}$ Synth. Met. 45 235-40 